\title{
Background Reduction in Cryogenic Detectors
}

\author{
Daniel A. Bauer \\ Fermi National Accelerator Laboratory
}

\begin{abstract}
This paper discusses the background reduction and rejection strategy of the Cryogenic Dark Matter Search (CDMS) experiment. Recent measurements of background levels from CDMS II at Soudan are presented, along with estimates for future improvements in sensitivity expected for a proposed SuperCDMS experiment at SNOLAB.
\end{abstract}

Keywords: Backgrounds, Cryogenics, Dark Matter, WIMPS

PACS: $95.30 . \mathrm{Cq}, 95.35 .+\mathrm{d}, 95.55 . \mathrm{Vj}$

\section{THE CRYOGENIC DARK MATTER SEARCH EXPERIMENT}

There are a large number of experiments trying to detect interactions of dark matter particles in terrestrial targets. One class of such experiments cools $\mathrm{Ge}$ and $\mathrm{Si}$ detectors to cryogenic temperatures $(<50 \mathrm{mK})$ in an attempt to discriminate the low energy $(<100 \mathrm{keV})$ nuclear recoils caused by dark matter WIMPS from the electron recoil interactions of conventional backgrounds. This paper discusses the background reduction strategy and measurements in one such experiment, the Cryogenic Dark Matter Search (CDMS II). The goal of the present incarnation of this experiment, CDMS II at Soudan, is direct direction of as few as 5 WIMP interactions per year, in the face of ambient backgrounds exceeding 10,000 events per year.

The CDMS II experiment operates at the Soudan Underground Laboratory (2070 mwe) in northern Minnesota. The rock overburden at Soudan reduces the cosmic ray muon flux to approximately 1 per minute hitting the experimental setup. Figure 1 shows elements of the CDMS II facility. The experiment is housed within an rf-shielded, class-10,000 cleanroom, with an auxiliary clean preparation area (anteroom), a room for electronics and data acquisition, and an area for cryogenic systems.


FIGURE 1. The CDMS II infrastructure at Soudan. Clockwise from upper left: the upper-half of the class-10,000 cleanroom which houses the experiment seen from the mezzanine level; the cryogenics system, with the dilution refrigerator and detector cold volume (icebox) connected via the cold stem; Towers $1 \& 2$ installed in the inner can of the icebox; a partial assembly of the shielding layers in the cleanroom. 
The experimental apparatus is hermetically surrounded by active shielding, consisting of two-inch-thick plastic scintillator panels. Inside of this "veto" is a $200-\mathrm{cm}$-tall by 200 -cm-diameter hollow cylindrical polyethylene shield with a 40-cm-thick top, bottom and wall, which moderates neutrons from surrounding materials. Inside of that is a 130$\mathrm{cm}$-tall by $130-\mathrm{cm}$-diameter hollow $\mathrm{Pb}$ shield with $22.5-\mathrm{cm}$ walls, which stops gamma rays produced by radioactivity in the rock. The inner $5 \mathrm{~cm}$ of this $\mathrm{Pb}$ is 'ancient', meaning that it contains very little of the long-lived ${ }^{210} \mathrm{~Pb}$ isotope. Farther inside is a second $10-\mathrm{cm}$-thick polyethylene shield, which moderates neutrons that penetrate the $\mathrm{Pb}$. Finally, at the center is the Icebox, a mostly-copper cryogenic chamber mapped to the thermal layers of the dilution refrigerator via a nested set of copper stems, (or cold stem). There is space in the base temperature $(<50 \mathrm{mK})$ chamber for seven six-detector "towers" arranged in a hexagonal pattern. The detector signals are brought out through another set of nested copper stems to a vacuum bulkhead and from there to front end electronics.

The CDMS ZIP (Z-dependent Ionization and Phonon) detector technology, using simultaneous athermal phonon and ionization measurement in Ge and Si crystals, has demonstrated outstanding rejection of gamma and beta backgrounds. These background particles scatter off electrons in the detectors, while WIMPs (and neutrons) scatter off nuclei. The ZIP detectors allow discrimination between electron and nuclear recoils through two effects. First, recoiling electrons are more ionizing than recoiling nuclei, resulting in a higher ratio of ionization to phonon signal, called "ionization yield." Figure 2 shows the discrimination based on ionization yield. Second, the athermal phonon signals due to nuclear recoils have longer rise times and occur later than those due to electron recoils, as is shown in Fig. 3. For recoils within a few $\mu \mathrm{m}$ of a detector's surface (primarily from low-energy electrons), the charge collection is less complete, making discrimination based on ionization yield less effective. Many of these events can be rejected by phonon timing cuts because they have even faster phonon signals than those from bulk electron recoils.

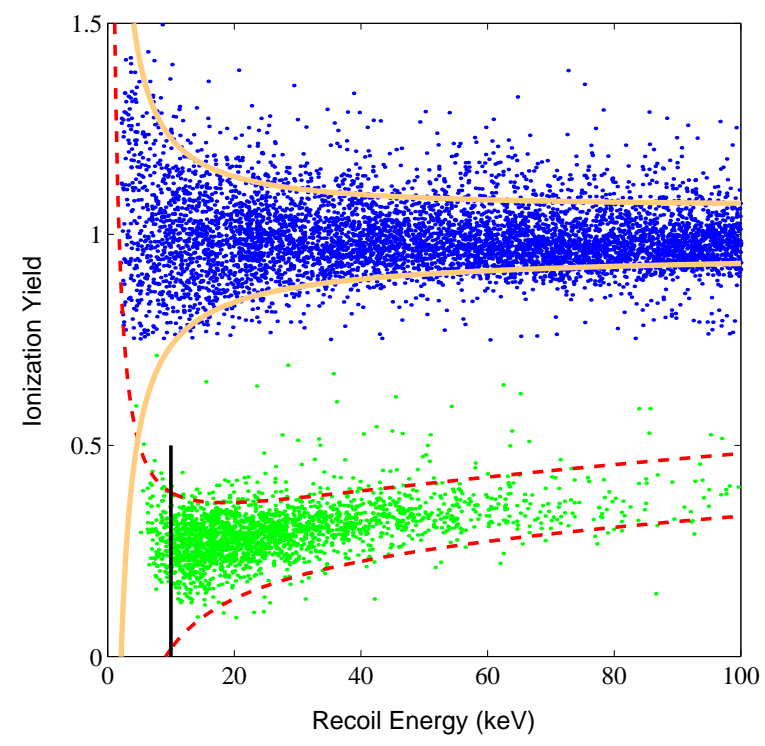

FIGURE 2. Ionization yield versus recoil energy for calibration data with a ${ }^{252} \mathrm{Cf}$ gamma and neutron source for Ge ZIP detectors showing the $\pm 2 \sigma$ gamma band (solid curves) and the $\pm 2 \sigma$ nuclear-recoil band (dashed curves). Events with ionization yield $<0.75$ (green) are shown only if they pass the phonon-timing cuts. The vertical line is the $10 \mathrm{keV}$ analysis threshold.

While neutrons cannot be distinguished from WIMPs on an event-by-event basis, because both produce nuclear recoils, they can be statistically separated in two ways. First, neutrons will frequently scatter in more than one detector, whereas WIMPs will not. Second, the cross sections for neutron interactions in Ge and Si are similar, but the (spinindependent) WIMP cross sections are coherent, scaling as $\mathrm{A}^{2}$, and are thus about 5 times larger in Ge than in Si.

\section{BACKGROUNDS}

All dark matter experiments must employ some form of shielding against backgrounds resulting from small amounts of radioactivity from the three naturally occurring chains ${ }^{238} \mathrm{U},{ }^{232} \mathrm{Th}$, and ${ }^{40} \mathrm{~K}$. These chains produce a mixture of gammas, betas, alphas, and neutrons with typical energies of a few MeV. Trace quantities of these isotopes are always present in the materials surrounding the experiment and great care must be taken to reduce the amounts present 


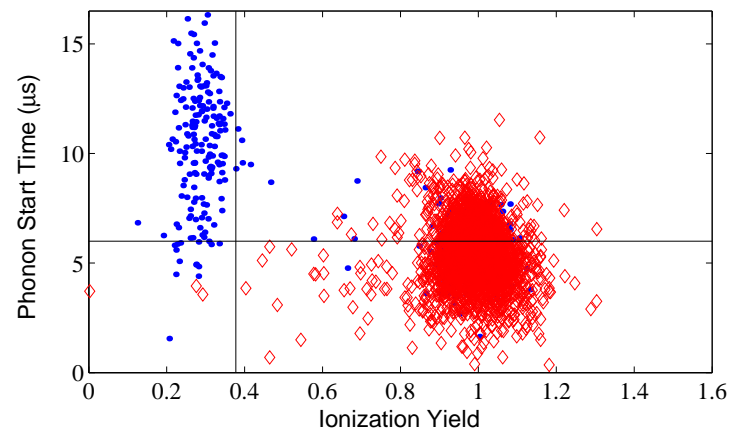

FIGURE 3. Phonon start time versus ionization yield for ${ }^{133} \mathrm{Ba}$ gamma-calibration events (diamonds) and ${ }^{252} \mathrm{Cf}$ neutroncalibration events (dots) in the energy range $20-40 \mathrm{keV}$ in a Ge detector. Lines indicate typical timing and ionization-yield cuts, resulting in high efficiency for nuclear recoils and a low rate of misidentified electron-recoil events.

in the shielding materials themselves. Neutrons from radioactivity arise primarily from fission decays and (alpha,n) reactions. In addition to radioactivity, cosmic ray interactions provide a significant source of potential background particles, requiring that the experiment be located deep underground to reduce the cosmic ray muon flux. The most difficult backgrounds are due to high energy neutrons from cosmic ray interactions, since neutrons lead to nuclear recoil interactions just as WIMPs do. Most experiments surround their detectors with an active veto, to detect cosmic ray muons that would interact in the shielding materials and produce neutrons. However, if the cosmic ray interacts in the surrounding materials, the active veto may not detect the resulting high energy neutron. These neutrons are very difficult to moderate with shielding and form an irreducible background at a given depth. Figure 4 illustrates these background sources, and the following sections detail their measured levels in CDMS.

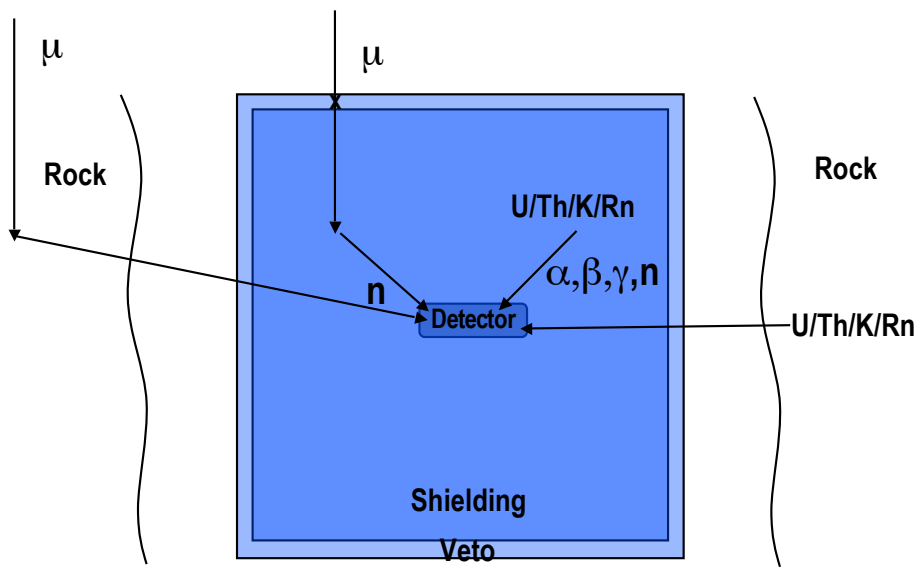

FIGURE 4. Conceptual diagram of background sources for dark matter experiments

\section{Electromagnetic Backgrounds}

The term "electromagnetic backgrounds" is used to refer to photons and electrons. Electromagnetic backgrounds divide into two subgroups, "bulk" and "surface" backgrounds, owing to the fact that the ZIP detectors possess a thin surface "dead layer" in which ionization-yield-based rejection is poor compared to that for bulk events. Events falling within the first $35 \mu \mathrm{m}$ suffer some ionization-yield suppression and events in the first $1 \mu \mathrm{m}$ lose so much ionization as 
to be misidentified as nuclear recoils. Phonon-timing-based rejection provides rejection of $97 \%$ of surface events, but the overall rejection of such events remains poorer than for bulk events.

Bulk electromagnetic backgrounds consist primarily of high-energy photons, as well as electrons emitted from radioisotopes in the bulk of the detectors. The photon component is determined by bulk contamination levels in the apparatus and shield and by radon penetration into the shield. Because the electron component is usually negligible in comparison to the photon component, bulk events are generically referred to as "photons." They are identified empirically as events with ionization yield $\approx 1$ as expected for bulk electron recoils.

Surface electromagnetic backgrounds include (1) "photon-induced" photons or electrons that interact in the dead layer and (2) electrons (and possibly low-energy X-rays) emitted by radioactive contaminants on detector or nearby surfaces. The photon-induced component scales directly with the continuum photon rate and detector surface area/volume ratio. Surface contamination depends on the detailed fabrication and handling history of the detectors. Surface events are generically referred to as "electrons." Surface events are identified empirically as all events with ionization yield below the "bulk" event band and above the nuclear-recoil band.

The rates of bulk and surface electromagnetic background events for Towers 1 and 2 are shown in Table 1.

TABLE 1. Average bulk and surface electromagnetic background rates in Tower 1 and Tower 2 at Soudan, 15$45 \mathrm{keV}$ deposited energy, inner detectors.

\begin{tabular}{|c||c|c|}
\hline event type & $\mathrm{Ge}$ & $\mathrm{Si}$ \\
\hline Bulk total & 115.2 & 501.9 \\
[/kg/day] & \pm 1.6 & \pm 4.6 \\
\hline Bulk singles & 30.2 & 194.5 \\
[/kg/day] & \pm 0.8 & \pm 2.3 \\
\hline Surface total & 1.57 & 1.53 \\
[/day] & \pm 0.09 & \pm 0.09 \\
\hline Surface singles & 0.41 & $\begin{array}{c}0.51 \\
\text { [/day] }\end{array}$ \\
\hline
\end{tabular}

Detector rejection for bulk electromagnetic backgrounds is extremely good. Rejection of events where a photon ejects an electron from the detector surface is similarly excellent, except for a negligible fraction of such events induced in the first $\mu \mathrm{m}$ of the surface. On the other hand, "electron" events from surface radioactive contaminants have a very shallow interaction depth profile and thus suffer far worse misidentification. About $0.6 \%$ of the surface single electrons in Table 1 survive to the nuclear-recoil signal region, contributing the dominant Soudan background of $1 \times 10^{-2} / \mathrm{kg} /$ day.

\section{Photon Backgrounds at Soudan}

We have simulated the contributions to the photon background from the $\mathrm{U} / \mathrm{Th} / \mathrm{K}$ content of the $\mathrm{Cu}$ cans, of the inner and outer polyethylene shields, and from ${ }^{210} \mathrm{~Pb}$ in the inner lead shield, with the simulations normalized by high purity $\mathrm{Ge}$ (HPGe) spectrometer screening results. The sum of these normalized contributions yields a photon background smaller by about a factor of 4 than the observed background, as shown in Figure 6.

A likely additional source of photons are radon decays outside the currently purged volume. The measured radon levels in the Soudan laboratory in the CDMS experimental room show a mean of about $500 \mathrm{~Bq} / \mathrm{m}^{3}$, with large seasonal variations, as shown in Figure 5. During early running at Soudan, before the radon purge was established, photon rates were a factor of 4-6 higher than shown in Table 1 and the ${ }^{214} \mathrm{Bi}$ radon daughter line at $609 \mathrm{keV}$ was clearly identified in the Ge detectors.

To reduce radon gas in the space directly surrounding the icebox, we are purging this volume with medical grade breathing air stored at least two weeks in metal cylinders to allow its radon to decay ( $\mathrm{T}_{1 / 2}=3.2$ days). However, the purge system in use when the data presented in Table 1 were taken did not penetrate the full volume, so significant radon buildup was possible outside the purged volume. Monte Carlo simulations show that about $35 \mathrm{~Bq} / \mathrm{m}^{3}$ of radon 


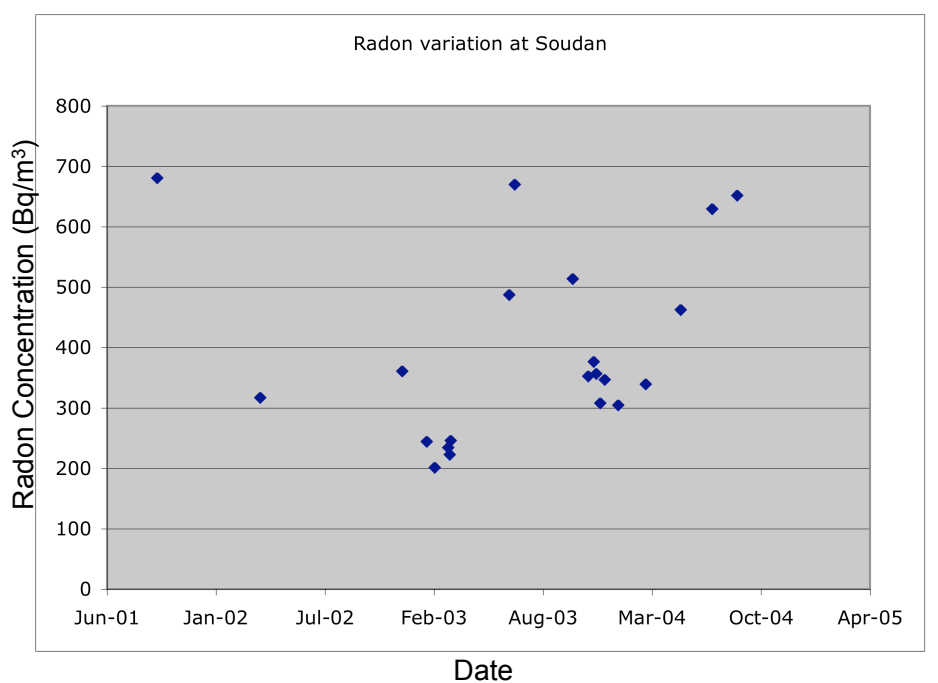

FIGURE 5. Measurements of Radon concentration in the air of the Soudan Underground Laboratory using a Rad7 counter made by Durridge. Radon concentration peaks in the summer and is lowest in the winter.

gas outside the purged volume is sufficient to explain most of the remaining photon background, as shown in Figure 6. In preparation for further running at Soudan, the purge system has been improved greatly.

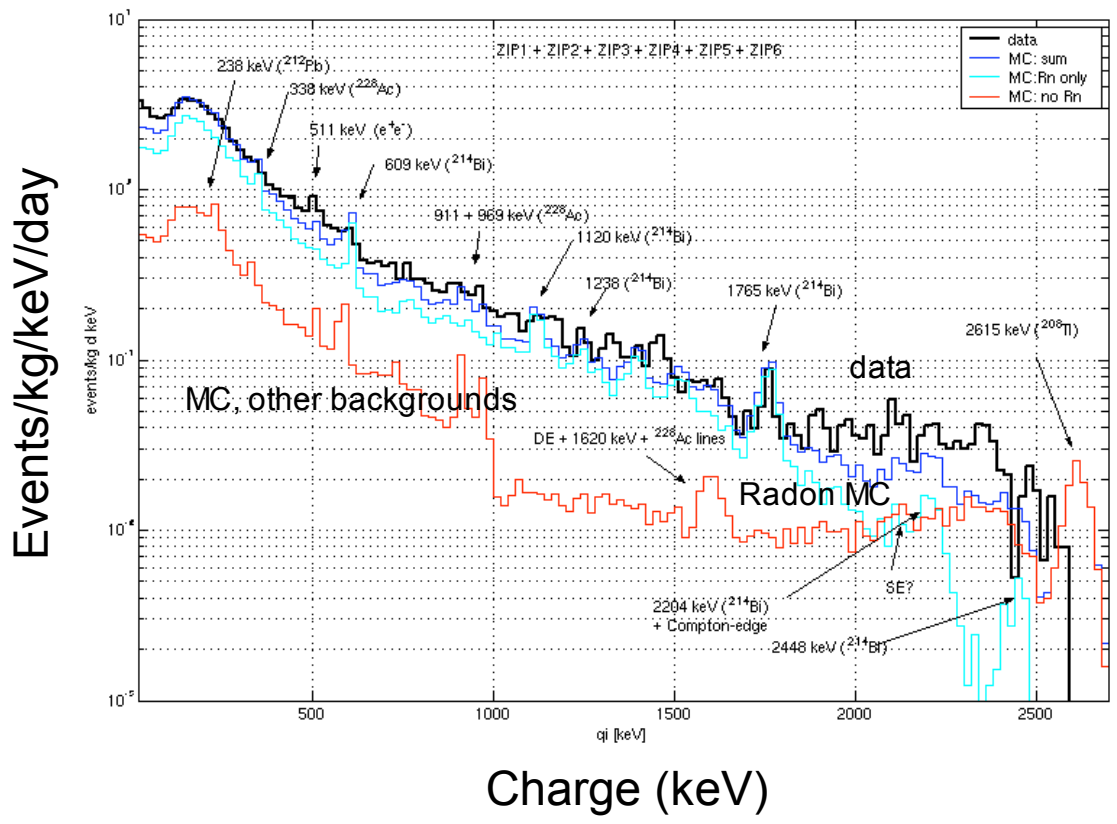

FIGURE 6. Measured gamma backgrounds in CDMS II detectors at Soudan compared with GEANT4 simulations incorporating known levels of radioactive elements in materials surrounding the detectors. Agreement between data and simulations is only achieved if significant Radon gas is present within the inner shielding of the experiment.

Rejection of photon-related backgrounds in the CDMS II experiment has been measured and simulated. Measurements are done by exposing the entire apparatus to high-energy photon sources via penetrations of the lead shield. Simulations are performed using GEANT4, including tracking of low-energy electrons created by photon interactions 
and conversion of interaction depth to ionization yield via the a yield vs. depth profile measured by exposure of a detector to a ${ }^{109} \mathrm{Cd}$ electron source.

The simulations indicate that of all photon-related events $(15-45 \mathrm{keV}), 0.31 \%$ are single-scatter surface events and $1.8 \%$ of those (or $0.005 \%$ of all photon-related events) suffer enough ionization-yield suppression to be misidentified as nuclear recoils. Thus, we have $99.995 \%$ rejection based on number of scatters and ionization yield alone. The singlescatter fraction is confirmed by photon-exposure calibration runs and by a comparison of pre/post-radon-purge rates. The overall misidentification is confirmed by seeing 1 single-scatter misidentified event in 25,000 photon-exposure events. The phonon-timing cut rejects low-yield events with $97 \%$ success, so the current overall rejection of photoninduced events is $99.9999 \%$. (The cuts have $70 \%$ exposure efficiency for nuclear recoils).

\section{Beta Backgrounds at Soudan}

The dominant CDMS background at Soudan consists of electrons from radioactive contamination on the detector surfaces, which we refer to as "betas," reflecting the likelihood that most of these events arise from beta decay. We include internal-conversion and Auger electrons as betas, and perhaps some soft x-rays as well. The total rate of surface electrons in the energy range $15-45 \mathrm{keV}$ is $\sim 1.6 /$ detector/day, of which $0.31 /$ detector/day are beta "singles," having a hit in one ZIP detector alone.

With a half-life $\mathrm{T}_{1 / 2}=22.3$ years, ${ }^{210} \mathrm{~Pb}$ is implanted into surfaces by a decay chain intiated by airborne ${ }^{222} \mathrm{Rn}$, which itself evolves from ${ }^{238} \mathrm{U}$. A total of $63 \mathrm{keV}$ is available in the transitions from ${ }^{210} \mathrm{~Pb}$ to the ${ }^{210} \mathrm{Bi}$ ground state, which liberates nearly one internal-conversion electron per decay with energy near $40 \mathrm{keV}$. The ${ }^{210} \mathrm{Bi}$ daughter with half-life $\mathrm{T}_{1 / 2}=5.01$ days undergoes $\beta^{-}$decay to ${ }^{210} \mathrm{Po}$ with $1.2 \mathrm{MeV}$ endpoint, but the $\beta$-electron energy distribution is non-standard, and peaks at the lowest energies. The ${ }^{210} \mathrm{Po}$ then decays with a half-life $\mathrm{T}_{1 / 2}=138$ days to the stable ${ }^{206} \mathrm{~Pb}$ isotope, emitting an $\alpha$ particle with an energy of $5.3 \mathrm{MeV}$.

Our detectors are exposed to radon during fabrication, mounting and testing. We have measured the ambient radon levels at all the locations where detectors travel and have taken precautions to store them in evacuated vessels or in cabinets purged with nitrogen gas for most of the time. The observed rate of $\alpha$ particles in our detectors is $\sim 0.4 /$ detector/day and suggests a similar contribution to the total rate of beta events. Further simulation work is in progress to determine what precise fraction of these events result in single-scatter events in the $15-45 \mathrm{keV}$ range.

Another beta-emitting candidate is ${ }^{40} \mathrm{~K}$, produced in the atmosphere by cosmic-ray interactions. Natural potassium may be deposited through human contact. The lack of a visible $1460 \mathrm{keV}$ photopeak or its Compton edge in the Soudan data sets an upper limit of 15 betas/day per ZIP over the full energy range up to the $1.4 \mathrm{MeV}$ endpoint, which corresponds to a limit on the ${ }^{40} \mathrm{~K}$ contribution to the total rate of betas of about 0.15 betas/day per ZIP in the $15-45 \mathrm{keV}$ energy region.

Stricter limits on the amount of ${ }^{40} \mathrm{~K}$ were set using materials-science surface-analysis techniques. Assays of test wafers produced at intermediate and final stages of processing were performed at campus facilities at Case Western Reserve University and the University of Minnesota using Secondary-Ion Mass Spectroscopy, Auger spectroscopy, RBS, and PIXE. We found that Rutherford backscattering (RBS) combined with particle-induced X-ray emission (PIXE) techniques, done simultaneously for each test wafer, provided the most sensitive normalized test for the presence of light elements such as potassium. RBS is capable of measuring the depth profile of elements and PIXE resolves ambiguities.

Our preliminary upper limit on natural $\mathrm{K}$ is $1-2 \times 10^{14}$ atoms $/ \mathrm{cm}^{2}$; confusion with Ar makes interpretation of the tests challenging. Further studies are in progress to determine whether this upper limit holds generically for witness wafers at all stages of fabrication.

This contamination level would yield a beta rate of about $1-2 /$ detector/day over all energies. Simulations indicate that about $8 \%$ of these betas produce energy depositions in the $15-45 \mathrm{keV}$ range and $15 \%$ of those (1.4\% overall) are single scatters, resulting in 0.02-0.04 single scatters/detector/day, a negligible contribution.

Our work with the above technique has convinced us we can detect buried and surface $\mathrm{K}$ layers as small as $1-2 \times 10^{13}$ atoms $/ \mathrm{cm}^{2}$ when there is no confusion with Ar. Such confusion can be avoid by replacing the $\mathrm{Ar}$ atmosphere used during metal deposition with $\mathrm{He}$ or $\mathrm{Kr}$.

A third possibility is ${ }^{14} \mathrm{C}$, also produced by cosmic-ray induced reactions. Natural carbon could be introduced during processing, and traces of carbon are easily measurable by surface analysis methods. Auger and RBS both indicate that there are 2-3 monolayers of C present on the ZIP surfaces, consistent with exposure to air. Depth profiles with RBS at the carbon nuclear resonance show that there is no buried carbon at this level. This yields 0.3 betas/day per ZIP with a 
$156 \mathrm{keV}$ endpoint. The contribution to the single-scatter rate in the $15-45 \mathrm{keV}$ range is thus likely small, no more than $15 \%$.

Overall, then, while we have quantitative estimates or limits for contamination by many possible emitters, we do not have a full accounting. We summarize our current knowledge in Table 2.

TABLE 2. Surface-event contributions estimated as indicated in the text. Although photons induce about $25 \%$ of the surface singles, they induce less than $1 \%$ of the events misidentified as nuclear recoils.

\begin{tabular}{|c|c|c|c|}
\hline source & origin & $\begin{array}{c}\text { rate } \\
\text { (\#/day })\end{array}$ & $\begin{array}{c}\% \text { of } \\
\text { singles }\end{array}$ \\
\hline${ }^{210} \mathrm{~Pb}$ & $\begin{array}{c}\text { airborne } \\
\text { radon }\end{array}$ & $\begin{array}{c}<0.4 \\
\text { all }\end{array}$ & $<100 \%$ \\
\hline${ }^{14} \mathrm{C}$ & $\begin{array}{c}\text { natural } \\
\text { carbon }\end{array}$ & $\begin{array}{c}<0.05 \\
\text { all }\end{array}$ & $<15 \%$ \\
\hline${ }^{40} \mathrm{~K}$ & $\begin{array}{c}\text { natural } \\
\text { potassium }\end{array}$ & $\begin{array}{c}<0.02-0.04 \\
\text { singles }\end{array}$ & $<15 \%$ \\
\hline
\end{tabular}

To progress toward identifying untested contaminants, we have assembled a list of 79 beta-emitting and electroncapture isotopes and performed a preliminary analysis of the possible contribution of these isotopes to our background of surface electrons. Contamination by these isotopes can be potentially determined by mass spectrometry, $\gamma$ detection, $\alpha$ detection, or of course by detecting the electron itself.

With a commonly achieved ICP-MS sensitivity of 1 ppb, 17 beta-emitting isotopes could be detected. A better ICPMS sensitivity of 1 ppt, which has been achieved for some isotopes, could allow detection of 20 additional isotopes. We will work with ICP-MS labs to test for these 37 isotopes on the detectors and in substances used during fabrication. In particular, ICP-MS routinely provides sensitivity to ${ }^{238} \mathrm{U}$ at the 100 part-per-trillion (ppt) level, sufficient for our needs.

Forty-three isotopes may be detected by low-level $\gamma$-counting, including 25 that cannot be detected by ICP-MS or $\alpha$-counting. $\gamma$ detection is advantageous for screening certain materials where high sensitivity can be achieved by using a large mass of sample. An upgrade of the HpGe (SOLO) $\gamma$ counting facility at Soudan, combined with a lowerbackground environment provided as part of the Soudan Low Background Counting Facility, will provide sensitive $\gamma$ screening.

A small number of isotopes, 12, can be detected by their emission of $\alpha$ 's. Although few in number, a crucial candidate, ${ }^{210} \mathrm{~Pb}$, is among these isotopes. We have already operated a small $\mathrm{Si}(\mathrm{Li})$ detector for $\alpha$ detection, but we believe that detector has a background level that is higher than desired for detecting $\alpha$ 's at the small flux now required. We plan to establish an $\alpha$ screening facility at the Soudan lab capable of detecting $\alpha$ 's of a flux $<5 \times 10^{-4} / \mathrm{cm}^{2} / \mathrm{d}$.

Finally, there are 12 to 21 isotopes, depending on ICP-MS sensitivity, that cannot be screened in any manner except by their emission of beta electrons. Direct counting of beta particles from large numbers of witness samples is a feasible method by which to detect unscreened isotopes. A sufficiently large screening chamber (or multiple chambers) can observe beta emission from a large surface area, of order 1 square meter, which is equivalent to 100 detectors. Witness samples can be run through the same fabrication and handling process as full detectors at a fraction of the manpower and cost. Rates can be measured quickly and feedback provided in a timely fashion.

However, such a large-area, low-background beta-screening device does not exist. We are beginning to develop it by prototyping two different technologies. The first is a neon-gas multi-wire proportional chamber, the second a cloud chamber read out by digital video. Both are optimized for identification and meausurement of sub-200 keV electrons. With appropriate construction materials, the limiting background in these detectors is ejection of electrons from the sample surface by Compton-scattering photons. In an environment that would yield a $1 / \mathrm{kg} / \mathrm{keV} / \mathrm{d}$ photon rate in Ge, the rate of such events is about $0.3 / \mathrm{m}^{2} / \mathrm{keV} /$ day.

A beta screener is not just useful for detecting betas, but makes an excellent alpha-particle counter as well. This ability provides another handle on many of the isotopes that could give rise to a beta background. Also, we can use the beta screener to check in detail our simulations of surface events. A contaminated detector could be run in a test facility to measure the observed spectrum of beta events in energy, yield, and phonon timing, and then the same detector could be placed in a beta screener. 
Our current level of beta rejection is estimated to be $99.4 \%$ rejection. The combination of ${ }^{109} \mathrm{Cd}$ calibration and WIMP-search data and simulations indicates that the ionization-yield-based rejection is about $80 \%$ for such events because of their shallow depth. The phonon-timing based rejection is $97 \%$ at $70 \%$ cut efficiency. The phonon-timing analysis is under active development and we are confident that at least a factor of five improvement in the beta rejection is possible. It would also be possible for future running to add 'ancient' $\mathrm{Pb}$ shielding around the detectors in the icebox to reduce both gamma and beta rates, as was done in CDMS I at the Stanford Underground Facility. Finally, we are considering the use of ionization-only 'endcap' detectors on our towers, to veto gammas and betas from outside materials.

\section{Neutron Backgrounds}

A neutron of kinetic energy approximately $2 \mathrm{MeV}$ can cause a Ge recoil that is indistinguishable from a recoil caused by a WIMP. A principal motivation for deploying WIMP searches at deep underground sites is the reduction of ambient cosmic-ray-induced neutron flux. Neutron background at Soudan arises from environmental radioactivity, primarily from processes in the Uranium/Thorium (U/Th) decay chain, and from particle cascades induced by muons that penetrate to Soudan's depth of approximately 2000 mwe. Neutrons from either process can originate in the material immediately surrounding the ZIP detectors ("internal") or from the relatively distant rock in the mine ("external"). Indeed, the radioactivity in the rock necessitates the massive CDMS II shielding consisting of $50 \mathrm{~cm}$-thick polyethylene to degrade the energy of neutrons from external radioactivity, and of $22.5 \mathrm{~cm}$ of lead to stop gammas from the same source. The polyethylene suppresses the rate of nuclear recoils due to neutrons from external radioactivity to insignificance. Selection of clean materials for the inner shield and icebox keeps the background from internal contaminations of U/Th small.

The concentration of shielding material near the ZIPs forms a target in which penetrating particles initiate showers of particles, including neutrons. We estimate the rate of Ge nuclear recoils (in the $15-45 \mathrm{keV}$ region) from neutrons in muon-induced showers in the shielding to be $18 \times 10^{-3} / \mathrm{kg} / \mathrm{day}$, with an uncertainty of a factor of 2 . Currently, we suppress this background with a hermetic, active, plastic scintillator veto system that surrounds the Soudan ZIP detectors. The Soudan veto system tags the incoming muon that initates showers in the shielding, with efficiency well in excess of 99\%, reducing this background to insignificance. In our published exposure [1], no neutrons were observed, where we would have expected $<0.05$ unvetoed neutrons and 1.9 vetoed neutrons. The full CDMS II exposure should constrain the rate of the neutron background with a fractional statistical error of $25 \%$.

The dominant source of neutrons that cause nuclear recoils in $\mathrm{Ge}$, and are anticoincident with the veto, come from showers produced by the $\sim 220 \mathrm{GeV}$ muons that penetrate to and interact in the rock that surrounds the CDMS II experimental hall. This muon-induced neutron background was estimated independently using two Monte Carlo techniques (GEANT4 and FLUKA) and checked for consistency.

These simulations indicate that associated shower particles produce signals in our existing veto for $60 \pm 10 \%$ of events containing neutrons. This implies a rate for the veto-anticoincident neutron-induced recoils from showers in the rock at Soudan of $3 \times 10^{-4} / \mathrm{kg} / \mathrm{day}$; small contributions from radioactivity raise the estimated total rate of background neutron-induced recoils to $4 \times 10^{-4} / \mathrm{kg} /$ day, with an uncertainty of a factor of 2 .

This background rate is sufficiently low that neutron background should be unimportant for the exposures currently planned at Soudan, but eventually they would limit further sensitivity improvements there. That is the principal motivation for proposing a larger version of CDMS (called SuperCDMS) to be located at SNOLab, where the dominant neutron backgrounds are suppressed compared to Soudan by over two orders of magnitude (see Figure 7).

One method to improve neutron rejection would rely on the detection of the neutrons themselves. Studies done five years ago for the CDMS II proposal, as well recent studies by the UKDMC [2], indicate that up to one order of magnitude suppression of neutron-induced Ge recoils can be achieved by an internal veto, just outside the Icebox. A schematic of an internal veto is shown in Fig. 8. The main role of the inner veto is to detect dangerous neutrons in the $\mathrm{MeV}$ energy range, via the recoil protons, with threshold of order $100 \mathrm{keV}$. Our current simulations indicate that upgraded veto systems at Soudan could extend background-free CDMS II running there to as much as $5000 \mathrm{~kg}$-d. 


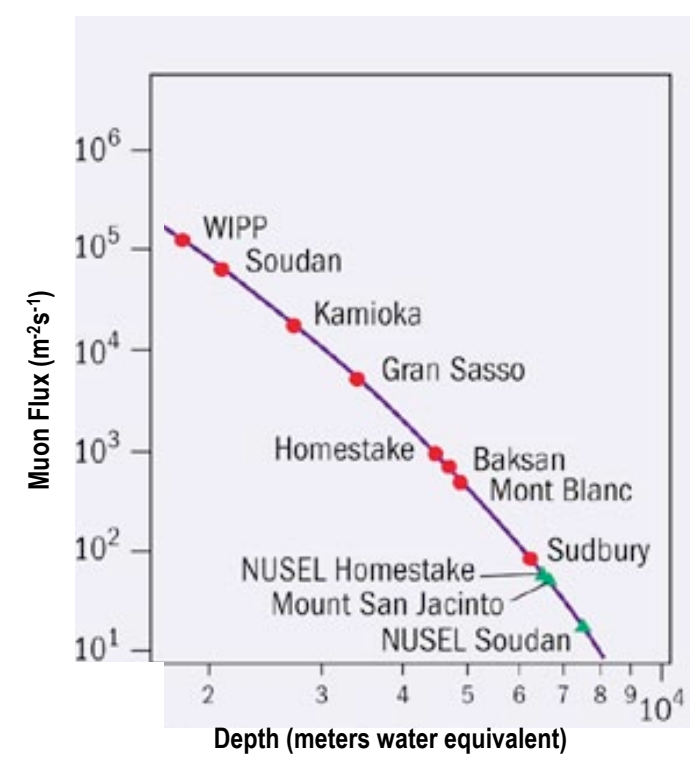

FIGURE 7. Muon flux as a function of depth in meters-water-equivalent, showing existing underground facilties.

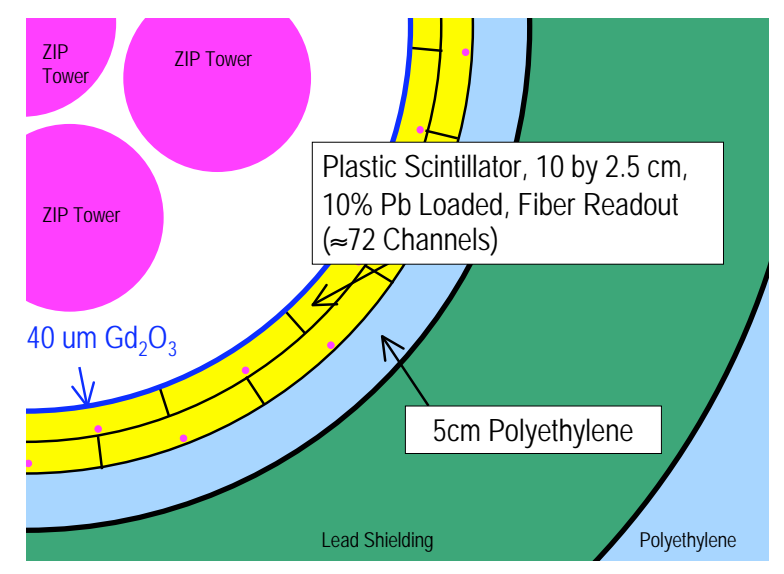

FIGURE 8. Possible Soudan Inner Veto. A double layer of plastic scintillator, read out by waveshifting fiber, would replace the inner $5 \mathrm{~cm}$ of the existing polyethylene shielding. Recoils of protons in the scintillator allow detection of MeV neutrons. A layer of $\mathrm{Gd}_{2} \mathrm{O}_{3}$ would capture slow neutrons, and emit about $8 \mathrm{MeV}$ in several photons, which would be detected in both the ZIP detectors and the scintillator. Only the "barrel" portion surrounding the ZIP detectors is shown; other, probably unsegmented "endcap" portions would go above and below the ZIP detectors.

\section{SCIENTIFIC REACH OF CDMS}

In the absence of backgrounds, the search sensitivity of a detector array is directly proportional to the mass $\times$ exposure time $(M T)$. In a mode where subtraction of an estimated background becomes necessary, the sensitivity improvement becomes proportional to $\sqrt{M T}$. Ultimately, the subtraction becomes limited by the systematics of calibrating the detector response to the background and no further improvement in sensitivity is possible. Thus, it is desirable to operate the experiment in "zero-background" mode to obtain the proposed large sensitivity gains.

The initial CDMS II results at Soudan [1] set an upper limit on the WIMP-nucleon cross-section of $4 \times 10^{-43} \mathrm{~cm}^{2}$ at the $90 \%$ C.L. at a WIMP mass of $60 \mathrm{GeV}$ for (spin-independent) coherent scalar interactions and a standard WIMP halo. In Figure 9 we show the expected progression of scientific reach through the end of running at Soudan and the possible reach of two possible SuperCDMS experimental phases at SNOLab. The dash-dot straight line corresponds 


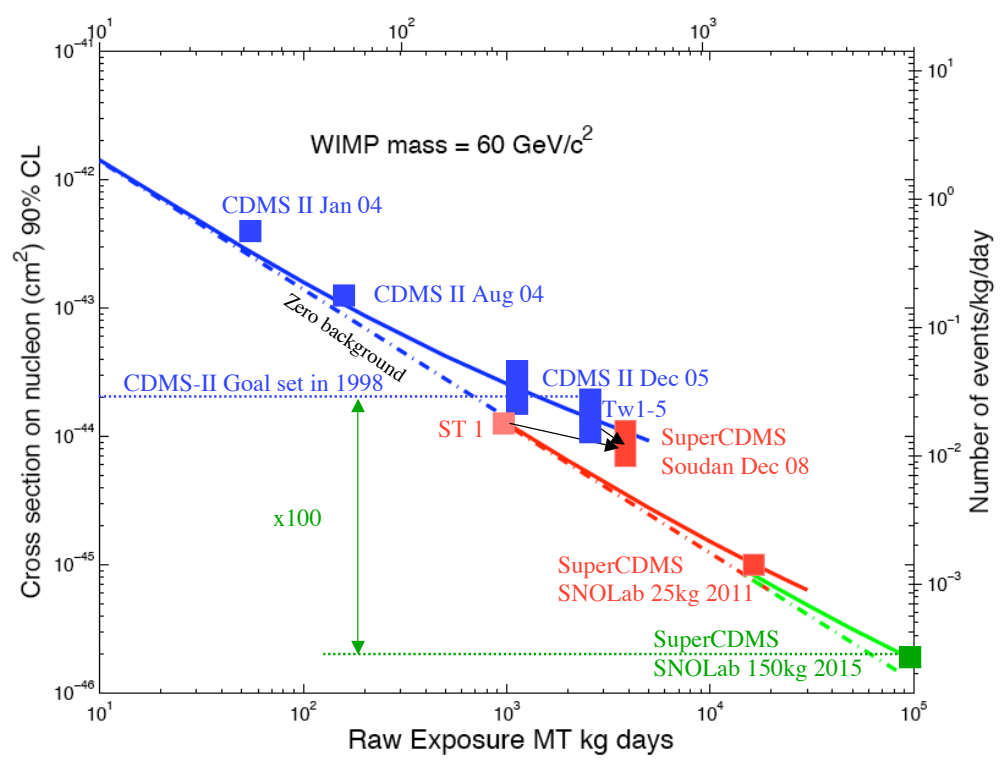

FIGURE 9. Limiting sensitivity $\left(\mathrm{cm}^{2}\right)$ as a function of exposure (kg-d) for CDMS II goals and SuperCDMS goals. These estimates use background subtraction and $10 \mathrm{keV}$ thresholds.

to the linear improvement in sensitivity for a zero background experiment. The curved lines show the effect of backgrounds which are subtracted after careful identification, and the rectangles are the projected reach at the end of each phase. Clearly the specific challenge for cryogenic detector experiments such as ours is to continue to improve beta rejection so as to stay near the zero background line.

\section{ACKNOWLEDGMENTS}

I would like to acknowledge all of my colleagues from the CDMS collaboration whose work is represented in this article. I would also like to thank all of the organizers of LRT2004 for a very interesting workshop and Richard Ford and Doug Hallman, in particular, for a fantastic tour of the SNO experiment.

\section{REFERENCES}

1. D.S. Akerib et al. (CDMS Collaboration), astro-ph/0405033, accepted by Phys. Rev. Lett.

2. P.F. Smith, Private communication. 